\title{
Linear Phase-gradient Imaging with Asymmetric Illumination based Differential Phase Contrast (AIDPC)
}

\author{
Shalin B. Mehta, ${ }^{1,2,3, *}$ and Colin J. R. Sheppard ${ }^{1,2,4}$ \\ ${ }^{1}$ Optical Bioimaging Lab, Division of Bioengineering, National University of Singapore, \\ Block-E3A, \#7-10, 7 Engineering Drive 1, Singapore 117574 \\ ${ }^{2}$ NUS Graduate School for Integrative Sciences \& Engineering (NGS), National University of Singapore \\ ${ }^{3}$ Liver Cancer Functional Genomics Lab, Humphrey Oei Institute of Cancer Research, National Cancer Center Singapore \\ ${ }^{4}$ Department of Biological Sciences, National University of Singapore \\ *Corresponding author: shalin@nus.edu.sg, shalin.mehta@gmail.com
}

\begin{abstract}
This paper demonstrates how one could engineer the transfer function of AIDPC, a novel optical thickness (phase) gradient measurement method suitable for live cell imaging, for linear imaging of the specimen phase-gradient.
\end{abstract}

OCIS codes: (110.4980) Partial coherence in imaging, (350.5030) Phase (c) 2009 Optical Society of America

\section{Introduction}

Asymmetric illumination based differential phase contrast (AIDPC) is a full-field phase-gradient imaging method derived from the scanning DPC system using the reciprocity theorem [1]. It is a quantitative method that offers significant advantages in the context of live cell imaging over currently popular methods of phase contrast and DIC [2,3]. Phase contrast restricts illumination aperture (uses condenser annulus) and modifies imaging aperture (phase ring in the objective) to achieve phase contrast, leading to reduced spatial resolution. It also suffers from halo effect preventing clear visualization of mitotic cells. DIC is a phase-gradient imaging method that allows imaging with large illumination and imaging apertures, providing good spatial resolution. However, DIC images a mix of cell morphology and light absorption. Moreover, it images phase gradient in a non-linear fashion [4,5]. Due to this non-linearity, retrieval of linear phase gradient from DIC images requires approaches such as phase-stepping [6-8], that need at least 3 images taken with different bias settings for single direction of shear. Retrieval of quantitative phase information requires measurement of the phase-gradient along at least two directions. Thus, linear imaging of phase with DIC requires at least 6 images, each of which requires mechanical movement of components which are hard to automate (e.g., changing the shear azimuth requires simultaneous rotation of two polarizers and two prisms or rotation of the sample). Our mathematical and experimental study of DIC [5] and AIDPC [2,3] indicates that AIDPC provides more quantitative measurement of phase-gradient information than DIC. As illustrated by Fig. 1, intensity in AIDPC image varies linearly with respect to the phase gradient. AIDPC requires acquisition of two images with opposite halves of the condenser aperture unlike three required by DIC for phase shifting. It is easy to automate acquisition of phase gradients in multiple directions with AIDPC, without any mechanical movement, using a spatial light modulator (SLM) as effective light source. Absence of mechanical vibrations ensures that images are acquired in precise registration (facilitating simpler image processing) and fast imaging speeds.

\section{Engineering linear relationship between specimen phase-gradient and image intensity}

Since AIDPC employs large illumination aperture, partially coherent image formation model is required to study its imaging properties $[9,10]$. The image intensity in a partially coherent system is given by,

$$
I(x, y)=\iiint \int T(m, n) T^{*}(p, q) C(m, n ; p, q) \exp [2 \pi j\{(m-p) x+(n-q) y\}] \mathrm{d} m \mathrm{~d} n \mathrm{~d} p \mathrm{~d} q,
$$

where $T(m, n)$ is the specimen's frequency spectrum, $T^{*}$ is the conjugate of the spectrum, $C(m, n ; p, q)$ is the partially coherent transfer function, $(m, p)$ are the pairs of frequency variables along the $X$-direction, and $(n, q)$ are the pairs 


\section{NTuA5.pdf}

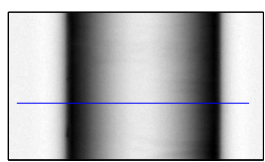

(a)

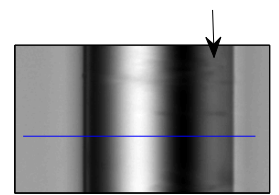

(b)

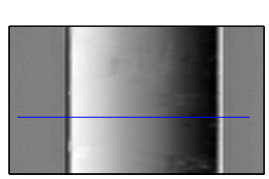

(c)

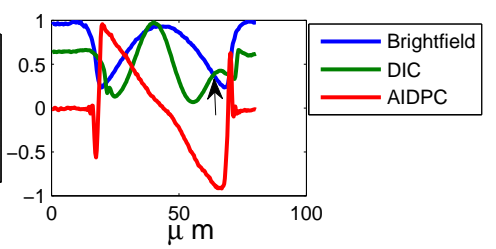

Fig. 1. AIDPC provides linear imaging of phase gradient: (a) Brightfield, (b) DIC (with as linear bias setting as possible), and (c)AIDPC images of an optical fiber (dia: $50 \mu \mathrm{m}$ ) aligned perpendicular to the differentiation azimuth. Traces through the images along the differentiation azimuth are shown. Optical fiber is a good test sample as it presents thickness gradients ranging from zero (at the center) to very large (at the edges) to the imaging system. Contrast in the brightfield image is only due to the steep gradients. While DIC provides useful visualization of the morphology of fiber, the relationship between the intensity and the gradient of optical thickness is highly nonlinear and can be artefactual (e.g., the arrow points to the contrast reversal artifact). AIDPC, on the other hand, provides artifact-free and linear imaging of the optical thickness gradient. Images were acquired with $20 \mathrm{X} 0.75 \mathrm{NA}$ objective with $0.75 \mathrm{NA}$ illumination. For fair comparison of contrast, all images were normalized by the highest intensity pixel in the image and displayed such that they occupy full dynamic range of the display.

of frequency variables along the $Y$-direction. Limits of integration in all the equations of this paper are from $-\infty$ to $\infty$. The partially coherent transfer function of the system can be computed as an area of overlap of three pupils as indicated by following equation.

$$
C(m, n ; p, q)=\iint\left|P_{\text {cond }}(\xi, \eta)\right|^{2} P_{o b j}(\xi-m, \eta-n) P_{o b j}^{*}(\xi-p, \eta-q) \mathrm{d} \xi \mathrm{d} \eta,
$$

where $P_{\text {cond }}$ is the condenser pupil and $P_{o b j}$ is the objective pupil. $\xi$ and $\eta$ are transverse co-ordinates in the pupil plane normalized with respect to $\mathrm{NA}_{o b j} / \lambda$.

Let us assume a specimen varying slowly with respect to the resolution of the imaging system (e.g., an optical fiber). If the gradient of the specimen thickness is $\psi_{\alpha}$ in the $X$-direction and $\psi_{\beta}$ in the $Y$-direction around region centered at co-ordinates $(x, y)=(\alpha, \beta)$, the specimen's transmission function can be approximated (within that region) by $\exp \left[2 \pi i\left(\psi_{\alpha} x+\psi_{\beta} y\right)\right]$. Consequently the spectrum can be approximated by $\delta\left(m-2 \pi \psi_{\alpha}\right) \delta\left(n-2 \pi \psi_{\beta}\right)$ around that region. From Eq. 1, we observe that the image intensity in such a case will be given by, $C\left(2 \pi \psi_{\alpha}, 2 \pi \psi_{\beta} ; 2 \pi \psi_{\alpha}, 2 \pi \psi_{\beta}\right)$. One can express the specimen transmission at each point with gradients and consequently the image as appropriate values of $C\left(2 \pi \psi_{\alpha}, 2 \pi \psi_{\beta} ; 2 \pi \psi_{\alpha}, 2 \pi \psi_{\beta}\right)$. Therefore, one can name the $C(m, n ; m, n)$ slice of the partially coherent transfer function as 'phase gradient transfer function (PGTF)'. PGTF is not a transfer function in the conventional sense of a filter in the spatial frequency domain. Rather, it is a look-up table that determines the relative intensity transmitted by a specimen having certain phase-gradient. As the phase-gradient increases, it refracts light out of the objective aperture (vignetting), which causes drop in the value of the PGTF. In brightfield configuration, when the phase-gradient corresponds to sum of the numerical apertures of the objective and the condenser pupils (representing cut-off of the PGTF), the light is completely refracted out of the objective. From Eq. 2, we see that PGTF is a convolution of magnitudes of the objective and condenser pupils. We have implemented an algorithm to calculate the complete partially coherent transfer function for any transmission imaging system using Eq. 2 [5, Sec.4].

For linear imaging of phase-gradient in a direction, one should engineer a PGTF varying linearly with respect to the specimen's phase-gradient in that direction. The PGTF's shape can be engineered by controlling the objective and condenser pupils. To allow aberration free imaging at full resolution, we choose to manipulate only the condenser pupil. We assume the differentiation azimuth to be in $X$-direction and examine the PGTF in that direction, $C(m, 0 ; m, 0)$, with different condenser geometries. 


\section{NTuA5.pdf}
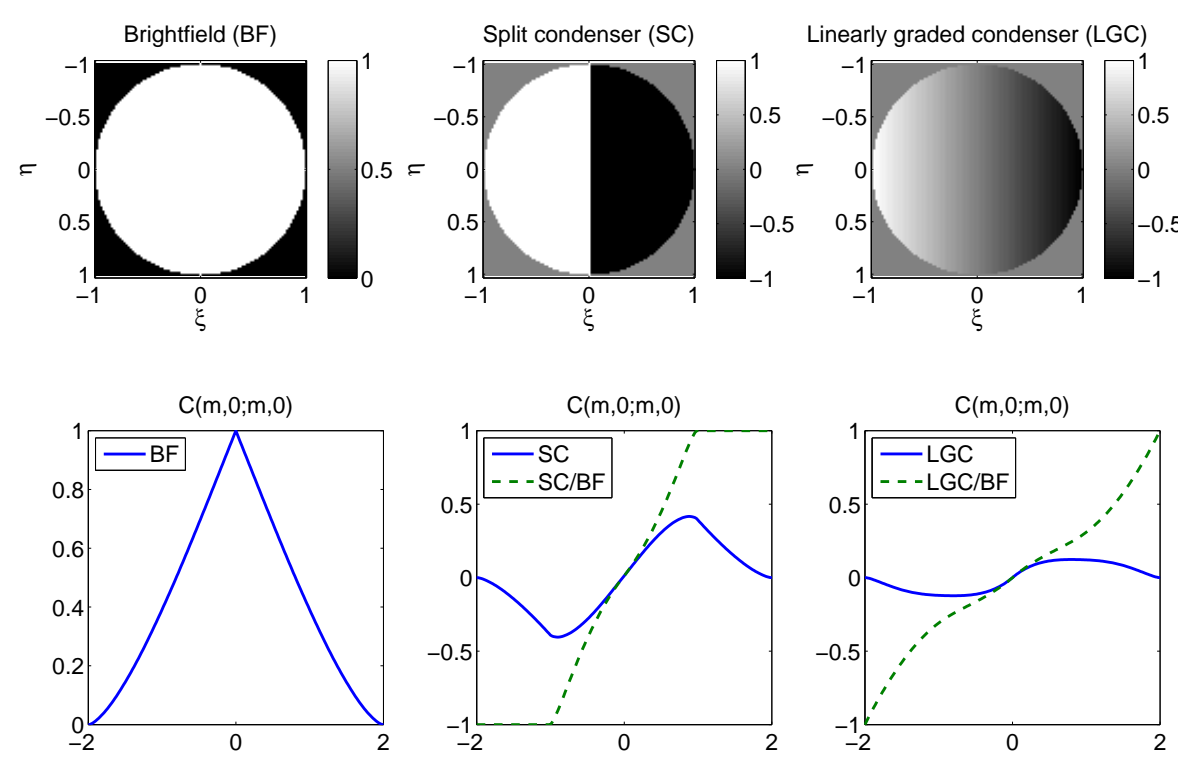

Fig. 2. Comparison of PGTFs (bottom row) obtained with different effective condenser pupils (top row). For all cases, the objective pupil is assumed to be a unit circle, i.e., same as the brightfield condenser pupil. The solid curves are the PGTF in the $X$-direction for condenser pupil geometry in top row. The dashed curves are the effective PGTFs for pixel-wise ratios of images acquired with pupils of top-row to the brightfield image.
Figure 2 shows the PGTF when circular (i.e., brightfield), semi-circular split (i.e. conventional DPC), or linearly graded condenser pupils are employed. The negative condenser pupil can be synthesized by acquiring images with two asymmetric pupils and subtracting the corresponding images. Since PGTF represents intensity, ratio of two PGTFs gives effective PGTF of the pixel-wise ratio of corresponding images. Fig. 2 illustrates that ratio of the image obtained with split circular pupil to the brightfield image has a nearly linear

effective PGTF up to half of the cut-off. On the other hand, ratio of the image obtained with linearly graded pupil to the brightfield image has a nearly linear effective PGTF up to the cut-off. This points to the possibility that optimization of the intensity structure of the illumination aperture in AIDPC will lead to linear phase-gradient imaging, and consequently to quantitative phase-retrieval at high spatial resolution.

C. J. R. Sheppard acknowledges support from the Singapore Ministry of Education Tier 1 funding (grants R397000022112 and R397000033112).

\section{References}

1. D. Hamilton and C. Sheppard, "Differential phase contrast in scanning optical microscopy," J. Microsc. 133(1), 27-39 (1984).

2. S. B. Mehta and C. J. R. Sheppard, "Asymmetric illumination based differential phase contrast (AI-DPC) for fullfield transmission imaging of phase information in biological specimens," , Focus on Microscopy 2008 (Awaji, Japan), http: //www. focusonmicroscopy.org/2008/PDF/089_Mehta.pdf.

3. S. B. Mehta, C. G. L. Lee, and C. J. R. Sheppard, "Quantitative phase-gradient imaging at high resolution with Asymmetric Illumination based Differential Phase Contrast (AIDPC)," (2009). Manuscript in preparation.

4. C. Cogswell and C. Sheppard, "Confocal differential interference contrast(DIC) microscopy: including a theoretical analysis of conventional and confocal DIC imaging," J. Microsc. 165, 81-101 (1992).

5. S. B. Mehta and C. J. R. Sheppard, "Partially coherent image formation in differential interference contrast (DIC) microscope," Opt. Express 16(24), 19,462-19,479 (2008).

6. M. R. Arnison, K. G. Larkin, C. J. R. Sheppard, N. I. Smith, and C. J. Cogswell, "Linear phase imaging using differential interference contrast microscopy," J. Microsc. 214(1), 7-12 (2004).

7. S. V. King, A. Libertun, R. Piestun, C. J. Cogswell, and C. Preza, "Quantitative phase microscopy through differential interference imaging," J. Biomed. Opt. 13(2) (2008).

8. C. J. Cogswell, N. I. Smith, K. G. Larkin, and P. Hariharan, "Quantitative DIC microscopy using a geometric phase shifter," Proceedings of SPIE 2984, 72 (1997).

9. C. J. R. Sheppard and A. Choudhury, “Image formation in the scanning microscope,” J. Mod. Opt. 24(10), 1051-1073 (1977).

10. H. H. Hopkins, "On the diffraction theory of optical images," Proc. R. Soc. Lond. A, Math. and Phys. Sci. 217(1130), 408-432 (1953). 